\title{
THE EFFECT OF TWO BODY CORRELATIONS ON THE CHARGE DENSITY DISTRIBUTIONS AND ELASTIC ELECTRON SCATTERING FORM FACTORS FOR SOME $2 s$-1d SHELL NUCLEI
}

\author{
A. K. Hamoudi, R. A. Radhi , G. N. Flaiyh and F. I. Shrrad \\ Department of Physics, College of Science University of Baghdad, Baghdad-Iraq.
}

\begin{abstract}
An effective two-body density operator for point nucleon system folded with two-body correlation functions, which take account of the effect of the strong short range repulsion and the strong tensor force in the nucleon-nucleon forces, is produced and used to study the ground state two-body charge density distributions $\rho_{c h}(r)$ and elastic electron scattering form factors $F(q)$ for $2 s-1 d$ shell nuclei with $Z=N$ (such as ${ }^{20} \mathrm{Ne},{ }^{24} \mathrm{Mg},{ }^{28} \mathrm{Si}$ and ${ }^{32} \mathrm{~S}$ nuclei). It is found that the inclusion of the two-body short range correlations $(S R C)$ has the feature of reducing the central part of $\rho_{c h}(r)$ significantly and increasing the tail part of $\rho_{c h}(r)$ slightly, i.e., it tends to increase the probability of transferring the protons from the central region of the nucleus towards its surface and to increase the root mean square charge radius $\left\langle r^{2}\right\rangle^{1 / 2}$ of the nucleus and then makes the nucleus to be less rigid than the case when there is no $S R C$. It is also found that the effects of two body tensor correlations $(T C)$ on $\rho_{c h}(r)$ and $\left\langle r^{2}\right\rangle^{1 / 2}$ are in opposite direction to those of $S R C$.
\end{abstract}

\section{Introduction}

The charge density distribution $\rho_{c h}(\mathrm{r})$ is one of the many most important quantities in nuclear structure which was well studied experimentally over a wide range of nuclei. This interest in $\rho_{c h}(\mathrm{r})$ is related to the basic bulk nuclear characteristics such as the shape and size of nuclei, their binding energies, and other quantities which are connected with $\rho_{c h}(\mathrm{r})$. Besides, the density distribution is an important object for experimental and theoretical investigations since it plays the role of a fundamental variable in nuclear theory [1]. The inclusion of short - range and tensor correlation effects is rather a complicated problem especially for the microscopic theory of nuclear structure. Several methods were proposed to treat complex tensor forces and to describe their effects on the nuclear ground state [2,3]. A simple phenomenological method for introducing dynamical short range and tensor correlations was presented by Dellagiacoma et al. [4]. In that method, a two-body correlation operator was introduced to act on the wave function of a pair of particles. A similar correlation operator was proposed earlier by Da Proveidencia and Shakin [5] as well as by Malecki and Picchi [6] for describing the short range correlation effects. The effect of the short range correlations due to the repulsive part of twobody interaction on the charge form factor of several p-shell nuclei had been analyzed in detail [7] with an independent particle model (IPM) generated in the harmonic oscillator (HO) well [8,9]. In Ref. [7], it was shown that the high-momentum parts $\left(q>3 \mathrm{fm}^{-1}\right)$ of the form factors calculated with and without correlations behave in completely different ways, which indicate that electron scattering at high momentum transfer could give useful information on the short-range correlations. The one-body charge density distributions of the ground state, elastic and inelastic electron scattering longitudinal form factors of $1 \mathrm{p}$-shell $[10,11]$ and 2 s-1d shell $[12,13,14]$ nuclei have been calculated with including the higher shells using the single particle wave functions of the harmonic oscillator potential. These studies have shown that introducing an additional parameter, that reflects the deviation of the occupation numbers of the states from the predictions of the simple shell model, leads to a very good agreement between the calculated and experimental results of the charge densities and elastic form factors. Core polarization effects $[10,11,13,14]$ have been considered in the calculations of the inelastic form factors where these effects are 
calculated using the one-body charge density distribution of the ground state through Tassie model [10]. These studies have found that core polarization effects are essential in reproducing a remarkable agreement between the calculated inelastic form factors and those of experimental data.

The cross-sections for elastic scattering of electrons from ${ }^{32} S$ nucleus were measured by Lombard et al. [15]. The elastic cross section was fitted well by a phase shift calculation for a static charge density. Bellicard et al.[16] reported experimental differential cross sections for the elastic scattering of electrons by ${ }^{40} \mathrm{Ca}$ and ${ }^{48} \mathrm{Ca}$ nuclei at $750 \mathrm{MeV}$. They compared their experimental results with those of the charge density distributions whose parameters were obtained from the $250 \mathrm{MeV}$ data. Traini and Orlandini [17] derived explicit expressions for $F(q)$ in the cases of ${ }^{4} \mathrm{He},{ }^{16} \mathrm{O}$ and ${ }^{40} \mathrm{Ca}$ taking into account the effects of short-range and tensor correlations within a phenomenological method for introducing dynamical short range and tensor correlations. They indicated that the effects of tensor correlations were more clearly shown for the light nuclei ${ }^{4} \mathrm{He}$ and ${ }^{16} \mathrm{O}$ than for ${ }^{40} \mathrm{Ca}$. The aim of the present work is to study the effects of short range correlations $f_{S C}(r)$ and tensor correlations $f_{T C}(r)$ on the ground state two body charge density distributions, root mean square charge radii and elastic electron scattering form factors for $2 s-1 d$ shell nuclei with $Z=N$, such as ${ }^{20} \mathrm{Ne},{ }^{24} \mathrm{Mg},{ }^{28} \mathrm{Si}$ and ${ }^{32} \mathrm{~S}$ nuclei.

\section{Theory}

The nucleon density of a nucleus consisting of $A$-point like particles is defined in terms of the one body density operator [1]

$$
\left.\hat{\rho}^{(1)}(\stackrel{r}{r})=\sum_{\mathrm{i}=1}^{\mathrm{A}} \delta(r) r_{r}^{r}\right)
$$

This operator can be transformated into a twobody density form as [18].

$$
\begin{aligned}
& \sum_{\mathrm{i}=1}^{\mathrm{A}} \delta\left(r-r_{r}^{r}\right) \equiv \frac{1}{2(A-1)} \sum_{i \neq j}\left\{\delta\left(\stackrel{r}{r}-r_{i}^{r}\right)+\right. \\
& \left.\delta\left(\stackrel{r}{r}-r_{j}\right)\right\}
\end{aligned}
$$

A further useful transformation can be made, which is that of the coordinates of the two particles $r_{i}$ and $r_{j}$ to be in terms of relative $r_{i j}$ and center of mass $\vec{R}_{\mathrm{ij}}$ coordinates [19]

$$
\begin{aligned}
& \stackrel{r}{r}_{i}=\frac{1}{\sqrt{2}}\left(\vec{R}_{\mathrm{ij}}+\stackrel{r}{r}_{i j}\right) . . \\
& r_{j}=\frac{1}{\sqrt{2}}\left(\vec{R}_{\mathrm{ij}}-\stackrel{r}{r}_{i j}\right)
\end{aligned}
$$

Using eqs. (3) and (4) in (2) yeilds

$$
\begin{aligned}
\hat{\rho}^{(2)}(r) & \frac{r}{2(A-1)} \sum_{i \neq j}\left\{\delta\left[\underset{i j}{r}-\frac{1}{\sqrt{2}}\left(R_{i j}^{r}+r_{i j}\right)\right]+\right. \\
& \left.\delta\left[\stackrel{r}{r}-\frac{1}{\sqrt{2}}\left(R_{i j}-r_{i j}\right)\right]\right\}
\end{aligned}
$$

Using the identity

$\delta(a \overrightarrow{\mathrm{r}})=\frac{1}{\left|a^{3}\right|} \delta(\overrightarrow{\mathrm{r}})$,

(where $a$ is a constant).Then eq. (5) can be written as

$$
\begin{gathered}
\left.\hat{\rho^{(2)}} \stackrel{r}{r}\right)=\frac{\sqrt{2}}{(A-1)} \sum_{i \neq j}\left\{\delta\left[\sqrt{2} \underset{r}{r}-R_{i j}^{r}-r_{i j}^{r}\right]+\right. \\
\left.\delta\left[\sqrt{2} \stackrel{r}{r}-R_{i j}+r_{i j}\right]\right\}
\end{gathered}
$$

An effective two-body charge density operator (to be used with uncorrelated wave functions) can be produced by folding the operator of eq. (6) with the two-body correlation functions $\tilde{f}_{i j}$ as

$$
\begin{aligned}
& \left.\hat{\rho}_{e f f}^{(2)} \stackrel{r}{r}\right)=\frac{\sqrt{2}}{2(A-1)} \sum_{\mathrm{i} \neq \mathrm{j}} \tilde{f}_{i j}\left\{\delta\left[\sqrt{2} \stackrel{r}{r}-\stackrel{r}{R}_{i j}-r_{i j}^{r}\right]+\right. \\
& \left.\delta\left[\sqrt{2} \stackrel{r}{r}-R_{i j}+r_{i j}^{r}\right]\right\} \tilde{f}_{i j}
\end{aligned}
$$

where the form of $\tilde{f}_{i j}$ is given by [20]

$\tilde{f}_{i j}=f\left(r_{i j}\right) \Delta_{1}+f\left(r_{i j}\right)\left\{1+\alpha(A) S_{i j}\right\} \Delta_{2}$

It is clear that eq. (8) contains two types of correlations:

1.The two body short range correlations presented in the first term of eq. (8) and denoted by $f\left(r_{i j}\right)$. Here $\Delta_{1}$ is a projection operator onto the space of all two-body wave functions with the exception of ${ }^{3} S_{1}$ and ${ }^{3} D_{1}$ states. It should be remarked that 
the short range correlations are central functions of the separation between the pair of particles which reduce the two-body wave function at short distances, where the repulsive core forces the particles apart, and heal to unity at large distance where the interactions are extremely weak. A simple model form of $f\left(r_{i j}\right)$ is given by [20]

$f\left(r_{i j}\right)=\left\{\begin{array}{cc}0 & \text { for } r_{i j} \leq r_{c} \\ 1-\exp \left\{-\mu\left(r_{i j}-r_{c}\right)^{2}\right\} & \text { for } r_{i j}>r_{c}\end{array}\right\}$

where $r_{c}$ (in $f m$ ) is the radius of a suitable hard core and $\mu=25 \mathrm{fm}^{-2}$ [20] is a correlation parameter.

2.The two-body tensor correlations presented in the second term of eq.(8) are induced by the strong tensor component in the nucleon-nucleon force and they are of longer range. Here $\Delta_{2}$ is a projection operator onto ${ }^{3} S_{1}$ and ${ }^{3} D_{1}$ states only. $S_{i j}$ is the usual tensor operator, formed by the scalar product of a second-rank operator in intrinsic spin space and coordinate space and is defined by [20]:

$S_{i j}=\frac{3}{r_{i j}^{2}}\left(\stackrel{r}{\sigma_{i}} \cdot r_{i j}\right)\left(\stackrel{r}{\sigma}{ }_{j} \cdot r_{i j}\right)-\stackrel{r}{\sigma_{i}} \cdot \stackrel{r}{\sigma}$

The parameter $\alpha(A)$ is the strength of tensor correlations and it is non zero only in the ${ }^{3} S_{1}-{ }^{3} D_{1}$ channels.

The ground state two body charge density distribution $\rho_{c h}(r)$ is given by the expectation value of the effective two-body charge density operator of eq. (7) and expressed as

$$
\begin{aligned}
& \rho_{c h}(r) \equiv\left\langle\Psi\left|\hat{\rho}_{\text {eff }}^{(2)}(\overrightarrow{\mathrm{r}})\right| \Psi\right\rangle= \\
& \sum_{i\langle j}\left\langlei j \left|\hat{\rho}_{e f f}^{(2)}(\overrightarrow{\mathrm{r}})[|i j\rangle-|j i\rangle]\right.\right.
\end{aligned}
$$

where the two particle wave function is given by [21] $|i j\rangle=\sum_{J M_{J} T M_{T}}\left\langle j_{i} m_{i} j_{j} m_{j} \mid J M_{J}\right\rangle$

$\left\langle t_{i} m_{t_{i}} t_{j} m_{t_{j}} \mid T M_{T}\right\rangle\left|\left(j_{i} j_{j}\right) J M_{J}\right\rangle\left|\left(t_{i} t_{j}\right) T M_{T}\right\rangle$

It is important to indicate that our effective two body charge density operator of eq (7) is constructed in terms of relative and center of mass coordinates, thus the space-spin part $\left|\left(j_{i} j_{j}\right) J M_{J}\right\rangle$ of the two-particle wave function constructed in $j j$-coupling scheme must be transformed in terms of relative and center of mass coordinates.

The nuclear mean square charge radius $\left\langle r^{2}\right\rangle$ is defined by [21]

$<r^{2}>=\frac{4 \pi}{Z} \int_{0}^{\infty} \rho_{c h}(r) r^{4} d r$

Elastic electron scattering form factor for spin zero nuclei is related to the ground state two-body charge density distribution $\rho_{c h}(r)$ of eq. (11). In the Plane Wave Born Approximation (PWBA), the incident and scattered electron waves are considered as plane waves and $\rho_{c h}(r)$ is real and spherically symmetric, therefore, the form factor is simply the Fourier transform of $\rho_{c h}(r)$ and is defined by $[22,23]$

$F(q)=\frac{4 \pi}{Z} \int_{0}^{\infty} \rho_{c h}(\mathrm{r}) j_{0}(q \mathrm{r}) \mathrm{r}^{2} d \mathrm{r}$

where $j_{0}(q \mathrm{r})=\sin (q \mathrm{r}) /(q \mathrm{r})$ is the zeroth order spherical Bessel function and $q$ is the momentum transfer from the incident electron to the target nucleus. Eq. (14) may be expressed as

$F(q)=\frac{4 \pi}{q Z} \int_{0}^{\infty} \rho_{c h}(\mathrm{r}) \sin (q \mathrm{r}) \mathrm{r} d \mathrm{r}$

The nucleon finite size correction $F_{f s}(q)$ is defined by [24]

$F_{f s}(q)=e^{-0.43 q^{2} / 4}$

where $F_{f s}(q)$ is considered as a free nucleon form factor and assumed to be the same for protons and neutrons.

The center of mass correction $F_{c m}(q)$ is defined by [24] 


$$
F_{c m}(q)=e^{q^{2} b^{2} / 4 A}
$$

where $F_{c m}(q)$ removes the spurious state arising from the motion of the center of mass when shell model wave function is used. Introducing these corrections into eq. (15), we obtain

$$
F(q)=\frac{4 \pi}{q Z} \int_{0}^{\infty} \rho_{c h}(\mathrm{r}) \sin (q \mathrm{r}) \mathrm{r} d \mathrm{r} F_{f s}(q) F_{c m}(q)
$$

Elastic electron scattering form factor $F(q)$ including the effect of two body correlation functions can now be calculated by introducing the ground state $\rho_{c h}(r)$ of eq. (11) into eq. (18).

\section{Results and Discussion}

The calculations for the ground state twobody charge density distributions (2BCDD's) $\rho_{c h}(r)$, the root mean square charge radii $\left\langle r^{2}\right\rangle^{1 / 2}$ and elastic electron scattering form factors $F(q)$ 's are carried out for ${ }^{20} \mathrm{Ne},{ }^{24} \mathrm{Mg}$, ${ }^{28} \mathrm{Si}$ and ${ }^{32} \mathrm{~S}$ nuclei. The higher states $2 s_{1 / 2}$ and $1 d_{3 / 2}$ are included in the calculations for $\left({ }^{20} \mathrm{Ne},{ }^{24} \mathrm{Mg},{ }^{28} \mathrm{Si}\right)$ and ${ }^{32} \mathrm{~S}$ nuclei, respectively. The occupation probabilities of these higher states are considered as free parameters. A choice for the single value of the hard core radius $r_{c}=0.5 \mathrm{fm}$ is adopted for all considered nuclei. The strengths of the tensor correlations $\alpha(A)$ are determined by fitting the calculated $\left\langle r^{2}\right\rangle^{1 / 2}$ with those of experimental data. All parameters required in the calculations of $\rho_{c h}(r),\left\langle r^{2}\right\rangle^{1 / 2}$ and $F(q)^{\prime} s$, such as the harmonic oscillator spacing parameter, $h \omega$, the occupation probabilities, $\eta$ 's, of the states and $\alpha(A)$, are presented in Table (1). It is clear from this table that the result for $\alpha(A)$ is a decreasing function with increasing the mass number A. Besides, the calculated $\left\langle r^{2}>_{\mathrm{r}_{c}=0.5, \alpha \neq 0.0}^{1 / 2}\right.$ including both effects of SRC (with $r_{c}=0.5 \mathrm{fm}$ ) and TC (with $\alpha(A) \neq 0$ ), are in very good agreement with those of experimental data [25]. The results for the dependence of $\rho_{c h}(r)$ (in $\mathrm{fm}^{-3}$ ) on $\mathrm{r}$ (in $f m$ ) for ${ }^{20} \mathrm{Ne},{ }^{24} \mathrm{Mg},{ }^{28} \mathrm{Si}$ and ${ }^{32} \mathrm{~S}$ nuclei are displayed in Figs. (1 and 2).

In Fig. (1), the dashed, dash-dotted and solid distributions are the calculated results of $\rho_{c h}(r)$ without including the effects of correlations $\left(r_{c}=0\right.$ and $\left.\alpha(A)=0\right)$, including only the effect of SRC $\left(r_{c}=0.5 \mathrm{fm}\right.$ and $\alpha(A)=0)$ and including only the effect of $T C$ ( $r_{c}=0$ and $\alpha(A) \neq 0$ ), respectively. It is clear that including only the effect of $S R C$ reduces significantly the central part $(0 \leq r \leq 2 \mathrm{fm})$ of $\rho_{c h}(r)$ and increases slightly the tail part of $\rho_{c h}(r)$, i.e., this effect increases the probability of transferring the protons from the central region of the nucleus towards its surface and thus makes the nucleus less rigid than the case when there are no correlations. Thus, an increase in the calculated $\left\langle r^{2}\right\rangle^{1 / 2}$ is expected when the effect of $S R C$ is included. It is obvious from this figure that the effect of $T C$ on $\rho_{c h}(r)$ and $\left\langle r^{2}\right\rangle^{1 / 2}$ is in opossite direction to that of $S R C$.

In Fig. (2), the the dashed and solid distributions are the calculated $\rho_{c h}(r)$ of ${ }^{20} \mathrm{Ne}$, ${ }^{24} \mathrm{Mg},{ }^{28} \mathrm{Si}$ and ${ }^{32} \mathrm{~S}$ nuclei without $\left(r_{c}=0\right.$ and $\alpha(A)=0)$ and with the effects of SRC and TC $\left(r_{c}=0.5 \mathrm{fm}\right.$ and $\alpha(A) \neq 0$ ) included, respectively. These distributions are compared with those of experimental data [25], denoted by dotted symbols. In ${ }^{20} \mathrm{Ne}$ and ${ }^{32} \mathrm{~S}$ nuclei, the dashed distributions deviate from the experimental data especially at small $r$. Introducing the effects of $S R C$ and $T C$ tends to remove these deviations from the region of small $\mathrm{r}$ as seen in the solid duistributions. In ${ }^{24} \mathrm{Mg}$ and ${ }^{28} \mathrm{Si}$ nuclei, the calculated $\rho_{c h}(r)$ is improved by considering the effects of $S R C$ and $T C$ as seen in the solid distributions. However, both the dashed and solid distributions overestimate the data at the central region of $\rho_{c h}(r)$ whereas beyond this region they agree very well with the data. 
Table (1)

Parameters which have been used in the calculations of the present work for the 2BCDD's, and elastic longitudinal $F(q)$ 's of all nuclei under study.

\begin{tabular}{|c|c|c|c|c|}
\hline Nucleus & ${ }^{20} \mathrm{Ne}$ & ${ }^{24} \mathrm{Mg}$ & ${ }^{28} \mathrm{Si}$ & ${ }^{32} S$ \\
\hline $\mathrm{h} \omega$ & 11.6 & 11.53 & 11.5 & 10.9 \\
\hline$\eta_{1 S_{1 / 2}}$ & 1 & 1 & 1 & 1 \\
\hline$\eta_{1 P_{3 / 2}}$ & 1 & 1 & 1 & 1 \\
\hline$\eta_{1 P_{1 / 2}}$ & 1 & 1 & 1 & 1 \\
\hline$\eta_{1 d_{5 / 2}}$ & 0.1917 & 0.5 & 0.783 & 1 \\
\hline$\eta_{2 S_{1 / 2}}$ & 0.425 & 0.5 & 0.65 & 0.7 \\
\hline$\eta_{1 d_{3 / 2}}$ & - & - & - & 0.15 \\
\hline$\alpha(A)$ & 0.085 & 0.084 & 0.081 & 0.075 \\
\hline$\left\langle\mathrm{r}^{2}\right\rangle_{\mathrm{r}_{c}=0, \alpha=0}^{1 / 2}$ & 2.962 & 2.980 & 3.095 & 3.255 \\
\hline$\left\langle\mathrm{r}^{2}\right\rangle_{\mathrm{r}_{c}=0.5, \alpha \neq 0.0}^{1 / 2}$ & 2.964 & 2.978 & 3.093 & 3.254 \\
\hline$\left\langle\mathrm{r}^{2}\right\rangle_{\text {exp. }}^{1 / 2}[25]$ & $2.992(25)$ & $3.08(5)$ & $3.086(18)$ & $3.248(11)$ \\
\hline
\end{tabular}



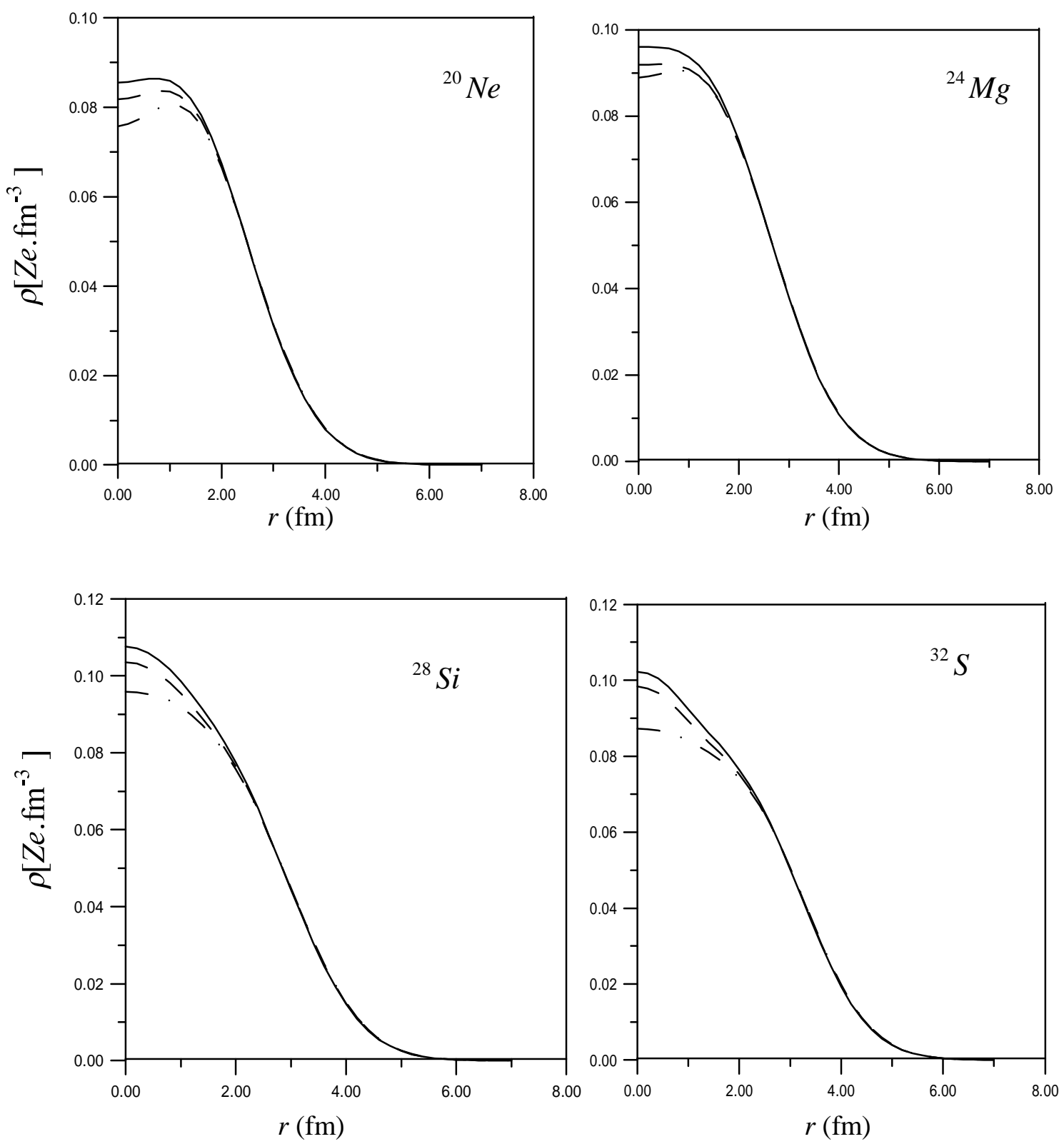

Fig.(1) : Dependence of the $2 \mathrm{BCDD}$ on $\mathrm{r}(\mathrm{fm})$ for ${ }^{20} \mathrm{Ne},{ }^{24} \mathrm{Mg},{ }^{28} \mathrm{Si}$ and ${ }^{32} \mathrm{~S}$ nuclei . 

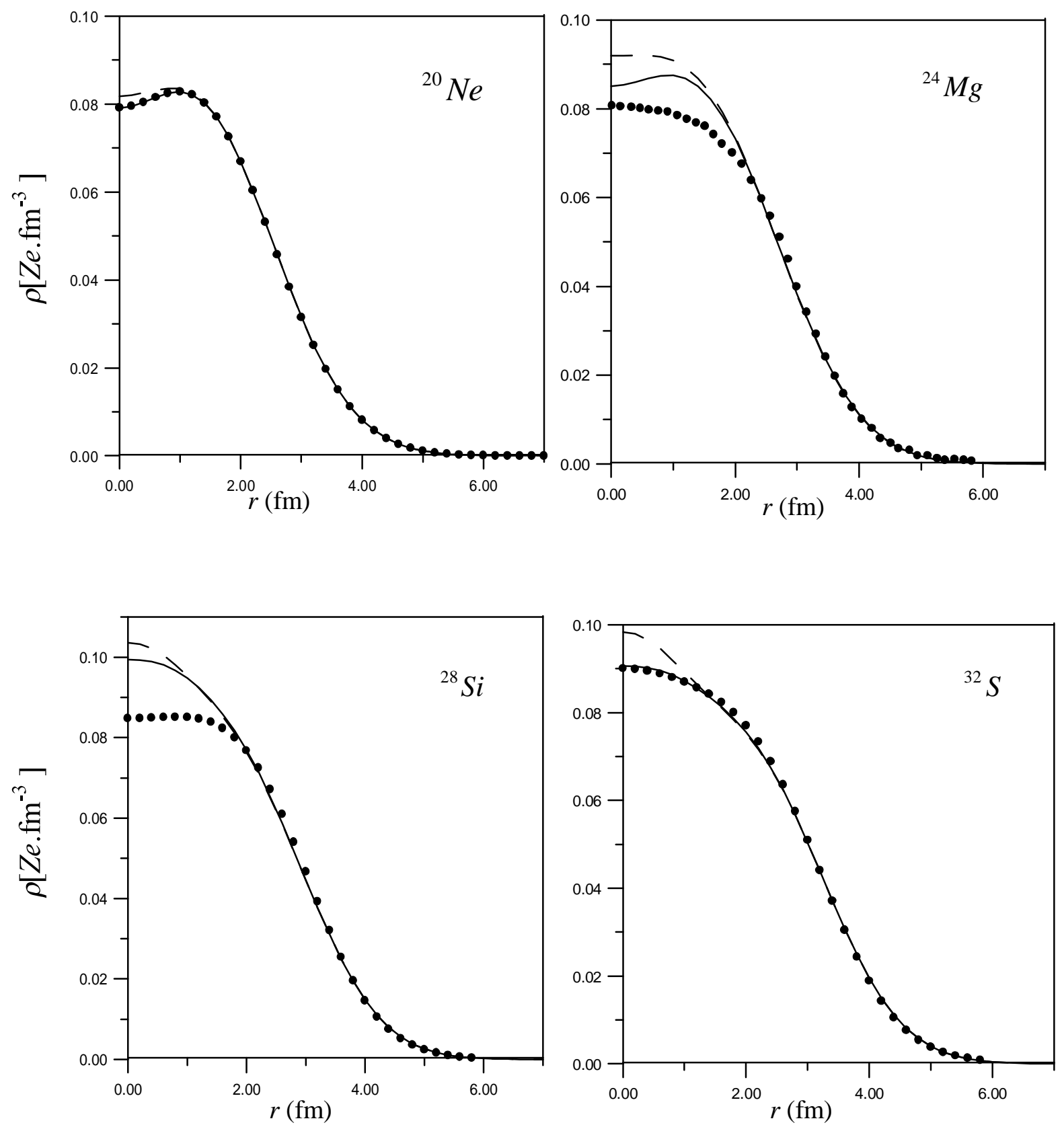

Fig.(2) : Dependence of the $2 \mathrm{BCDD}$ on $\mathrm{r}(\mathrm{fm})$ for ${ }^{20} \mathrm{Ne},{ }^{24} \mathrm{Mg},{ }^{28} \mathrm{Si}$ and ${ }^{32} \mathrm{~S}$ nuclei. The dotted symboles are the experimental data of Ref [25]. 
Elastic electron scattering charge form factors $F(q)$ for the considered 2 s- $1 d$ shell nuclei with $Z=N$ are calculated using the ground state two body charge density distributions of eq. (11) in eq. (18). In Fig.(3), the calculated $F(q)$ 's are compared with those of experimental data for ${ }^{20} \mathrm{Ne},{ }^{24} \mathrm{Mg},{ }^{28} \mathrm{Si}$, ${ }^{32} S$ nuclei. The dashed and solid distributions are the calculated $F(q)$ 's without and with the effects of $S R C$ and $T C$, respectively, while the dotted symbols are those of experimental data.

In ${ }^{20} \mathrm{Ne}$ nucleus, the available data are restricted for a small region of momentum transfer $q \leq 1.2 \mathrm{fm}^{-1}$. It is seen that the calculated $F(q)$ 's represented by the dashed and solid curves are in excellent agreement with those of experimental data [26].

In ${ }^{24} \mathrm{Mg}$ nucleus, the first diffraction minimum and first maximum which are known from the experimental data [27] are very well reproduced by the dashed and solid curves. In general, the calculated $F(q)$ 's are in very good accordance with the data up to momentum transfer $q \approx 2.4 \mathrm{fm}^{-1}$. For higher $q$, the calculated form factors are in disagreement with the data, where the second diffraction minimum observed in experiments is not reproduced in the correct place by the solid and dashed curves. It is noted that the effect of correlations begin at the region of $q>3 \mathrm{fm}^{-1}$, where the solid curve deviates from the dashed curve at this region of $q$.

In ${ }^{28} \mathrm{Si}$ and ${ }^{32} \mathrm{~S}$ nuclei, the first, second and third diffraction minima which are presented in the experimental data [27] are quite well described by the calculations of the solid curves. It is noted that the third diffraction minimum presented in the data and located at $q \approx 3.3 \mathrm{fm}^{-1}$ is not reproduced by the calculations of the dashed curve. However, the calculated $F(q)$ 's represented by, the dashed and solid curves clearly underestimate the data at the region of momentum transfer $q>1.2 \mathrm{fm}^{-1}$. It is evident that the inclusion of $S R C$ and $T C$ provides better description to the experimental data than the case when there are no correlations, where the behavior of the solid curves becomes in good accord with that of experimental data throughout the whole range of momentum transfer $q$. It is clear from this figure that the effect of the correlations becomes more effective at the region of momentum transfer $q>2.2 \mathrm{fm}^{-1}$.

\section{Conclusions}

This study shows that the two-body tensor correlations exhibit a mass dependence due to the strength parameter $\alpha(A)$ while the two-body short range correlations do not exhibit this dependency.
Including the effect of $S R C$ alone increases the probability of transferring the protons from the central region of $\rho_{c h}(r)$ towards its tail (i.e., the nucleus becomes less rigid than the case when there is no $S R C$ ) and then increases the calculated $\left\langle r^{2}\right\rangle^{1 / 2}$. It is found that the effect of $T C$ on $\rho_{c h}(r)$ and $\left\langle r^{2}\right\rangle^{1 / 2}$ is in opossite direction to that of $S R C$. It is seen that in the high momentum region $q>3 \mathrm{fm}^{-1}$ of elastic electron scattering, charge form factors $F(q)^{\prime} s$ calculated without and with the effects of $S R C$ and $T C$ behave in completely different ways, which indicates that the electron scattering at high momentum transfer can provide useful information on the $S R C$ and $T C$. 

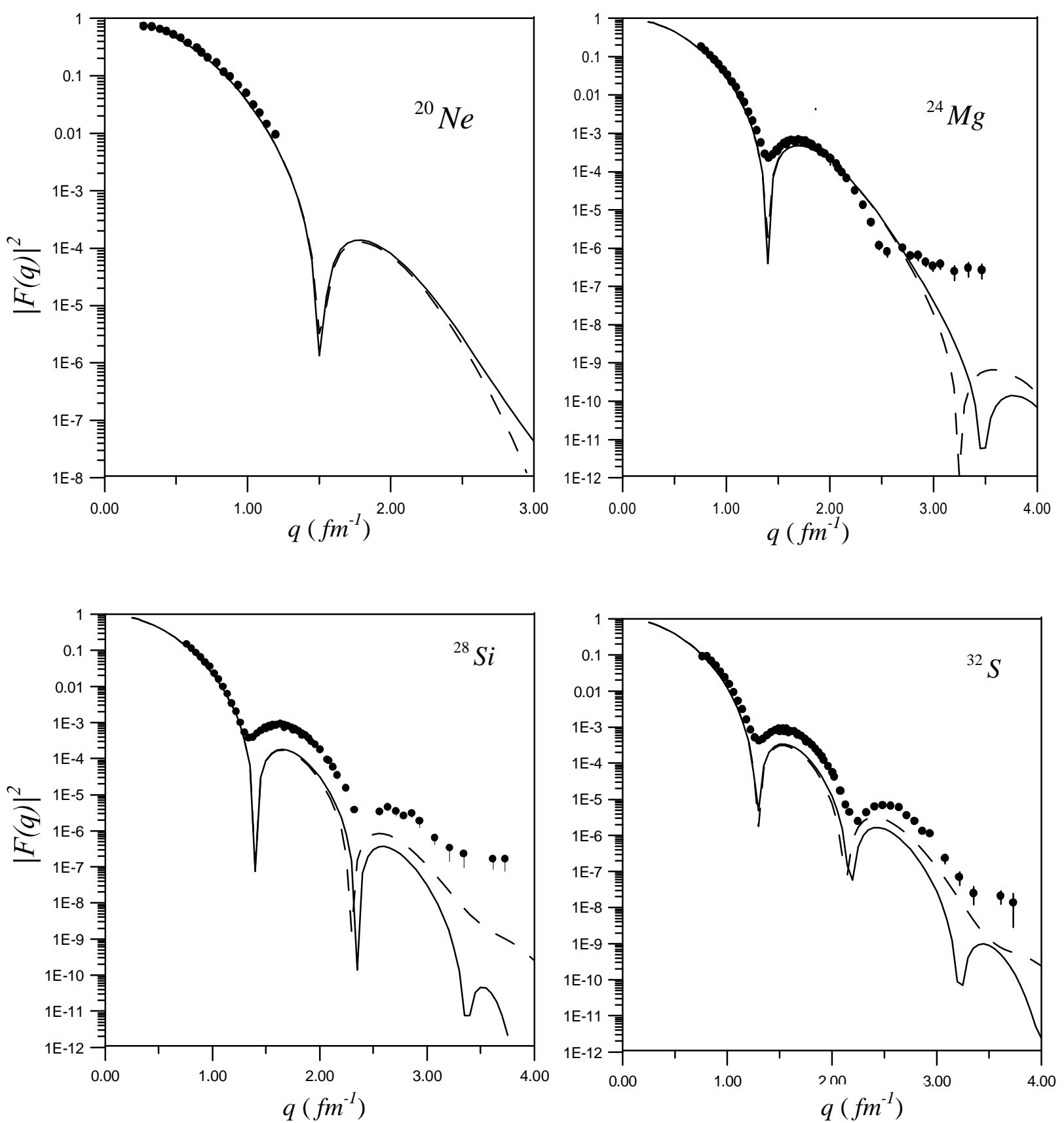

Fig.(3) : Dependence of the $\mathrm{F}(q)$ 's on $q\left(f m^{-1}\right)$ for ${ }^{20} \mathrm{Ne},{ }^{24} \mathrm{Mg},{ }^{28} \mathrm{Si}$ and ${ }^{32} \mathrm{~S}$ nuclei. The dotted symboles are the experimental data of Ref. [26] for ${ }^{20} \mathrm{Ne}$ and Ref.[27] for ${ }^{24} \mathrm{Mg}$, ${ }^{28}$ Si and ${ }^{32} S$ nuclei. 


\section{Refrences}

[1] A. N. Antonov, P. E.Hodgson and I. Zh. Petkov,(Nucleon Momentum and Density Distributions in Nuclei), Clarendon Press, Oxford, 1988, 97-102.

[2] H. Bethe, B. H. Brandow and. A. G. Petschek (Reference spectrum method for nuclear matter), Phys.Rev., 129, 1, 1963, 225-264.

[3] T. T. S.Kuo and G. E. Brown,(Structure of finite nuclei and the free nucleon-nucleon interaction), Nucl. Phys., A92, 3, 1967, 481-494.

[4] F. Dellagiacoma, G. Orlandiniand and M. Traini, ( Dynamical correlations in finite nuclei: A simple method to study tensor effects), Nucl. Phys.,A393, 1 , 1983, 95108.

[5] J. Da Providencia and C. M. Shakin, (Some aspects of short-range correlation in nuclei), Ann. Phys., 30, 1, 1964, 95-118.

[6] A. Malecki and P. Picchi, (Elastic electron scattering from ${ }^{4} \mathrm{He}$ ), Phys. Rev. Lett., 21, 19, 1968, 1395-1398.

[7] C. Ciofi Degli Atti, (Short range dynamical correlations in ${ }^{6} \mathrm{Li}$ from electron elastic scattering), Phys. Rev., 175, 4, 1968, 1256-1259.

[8] F. C. Khanna, (Nuclear structure of ${ }^{40} \mathrm{Ca}$ and elastic scattering of $750 \mathrm{MeV}$ electron), Phys. Rev. Lett., 20, 16, 1968, 871-873.

[9] C. Ciofi Degli Atti and N.M.Kabachnik, (Information on short range nucleonnucleon correlation and Wood-Saxon nuclear potentional from high energy elastic electron scattering), Phys. Rev., C1, 3, 1970, 809-815.

[10] Z. A. Salman, (Calculation of the longitudinal electron scattering form factors for the $1 \mathrm{p}$ - shell nuclei), M.Sc Thesis, University of Baghdad 2003.

[11] R. A. Radhi, A. K. Hamoudi and Z. A. Salman, (The calculation of the charge density distributions and the longitudinal form factors of ${ }^{10} B$ nucleus by using the occupation numbers of the states), Iraqi J. Phys., 2, 2003, 11-18.

[12] A. K. Hamoudi, A. A. Abdullah and E. M. Raheem, (Charge density distribution and elastic electron scattering form factors of $1 \mathrm{~d}-2 \mathrm{~s}$ shell nuclei using the occupation numbers of the states), Iraqi J. Sci, 45, 2004, 113-120.

[13] K. N. Flaih, (Calculation of the longitudinal electron scattering form factors for the $2 \mathrm{~s}-1 \mathrm{~d}$ shell nuclei using the ground state charge density distribution), M.Sc Thesis, University of Baghdad 2003.

[14] A. K. Hamoudi, R. A. Radhi and K. N. Flaih, (Inelastic longitudinal electron scattering form factors for the low mass $2 s$-1d shell nuclei), Proceeding of $3^{\text {rd }}$ Scientific Confference of College of Science, University of Baghdad, 2009.

[15] R. Lombard, P. Kossany and G. R.Bishop, ( The scattering of high energy electrons by ${ }^{32} \mathrm{~S}$ ), Nucl. Phys., A59, 1964, 398-406.

[16] J. B. Bellicard, P. Bounin, R. F. Frosch, R. Hofstadter, J. S. Mc Carthy, F. J. Uhrhane, M. R. Yearian, B. C. Clark, R. Herman, and D. G. Ravenhall, (Scattering of $750 \mathrm{MeV}$ electrons by calcium isotopes), Phys. Rev. Lett.,19,9, 1967, 527-529.

[17] M. Traini and G. Orlandini, (Nucleon momentum distributions in doubly closed shell nuclei), Z. Physik, A321 ,479, 1985, 479-484.

[18] S. Gartenhaus and C. Schwartz, (Center of mass in many particle systems), Phys. Rev., 108, 2, 1957, 482-490.

[19] R. D. Lawson, ( Theory of the Nuclear Shell Model), Clarendon Press, Oxford, 1980.

[20] J. Fiase, A. Hamoudi, J. M. Irvine and F. Yazici, ( Effective interaction for sd-shell model calculations) J. Phys, G14, 1, 1988, 27-36.

[21] Kris. L. G.Heyde, (The Nuclear Shell Model), Springer-Verlag Berlin Heidelberg, 1994,4-8.

[22] T. deforest, Jr. and J. D. Walecka, (Electron scattering and nuclear structure), Adv. Phys., 15, 1, 1966, 1-102.

[23] J. D. Walecka, (Electron Scattering for Nuclear and Nucleon Structure), Cambridge University Press, Cambridge, 2001, 14-16. [24] B. A. Brown, R. A. Radhi and B. H. Wildenthal, (Electric quadrupole and hexadecupole nuclear excitations from the perspectives of 
electron scattering and modern shellmodel theory), Phys. Rep., 101, 5, 1983, 313-358.

[25] H. De Vries, C. W. De Jager and C. De Vries, (Nuclear charge density distribution parameters from elastic electron scattering), Atomic data and nuclear data tables, 36, 3, 1987, 495-536.

[26] E. A. Knight, R. P.Singhal and M. W. Macauley; (Elastic scattering of electrons from $^{20} \mathrm{Ne}$ ) J.Phys., G7, 1981, 1115-1121.

[27] C. Li , M. R. Yearian and I. Sick; (High momentum-transfer electron scattering from ${ }^{24} \mathrm{Mg},{ }^{27} \mathrm{Al},{ }^{28} \mathrm{Si}$, and $\left.{ }^{32} \mathrm{~S}\right)$, Phys. Rev., C9, 1974, 1861-1877.

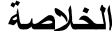

لقد تم توليد مؤثر الكثافة النوويــة الفعــال ذو صــيغة

الجسيمتين لنوى تكون فيها النويات بمثابة جسيمات نقطيــة

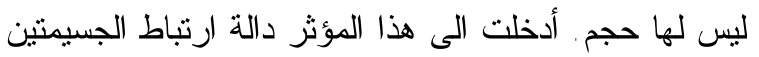

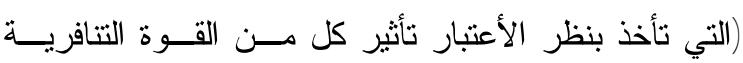

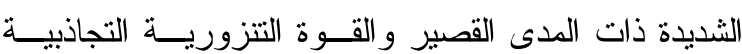

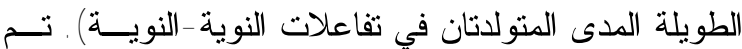

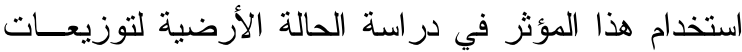

كثافة الثحنة النوويــة ذو صــيغة الجسيمتين

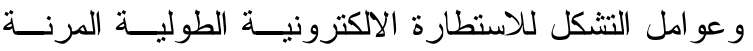

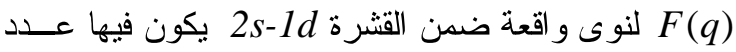

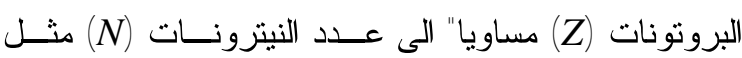
النوى

اظهرت هذه الدراسة بان ادخال دالة ارتباط الجسيمتين

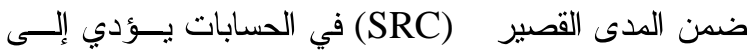

زيادة احتمالية انتقال البرونونات من مركز النـــــاة باتجـــاه

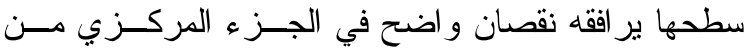

توزيعات كثافة الثحنة النووية (r)

الجزء ألذيلي منه وبالتالي فأن القيمة المحسوبة لجذر معدل

مربع نصف القطر

بأن تكون أقل صلادة من الحالة التي كانت فبها النواة بدون

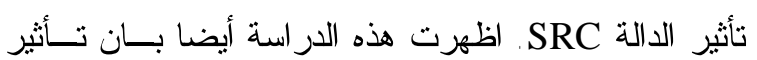

الدالة التنزورية لأرتباط الجسيمتين (TC) على كـل مسن التهن

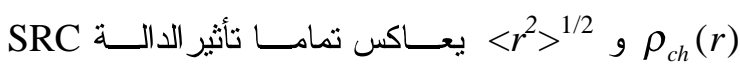

